\title{
Questionable Research Practices and Determinants of Their Frequency
}

\author{
B.Sc. Psychology thesis \\ Taym $^{1}$ Alsalti \\ Supervisor: Prof. Dr. Karl Christoph Klauer ${ }^{2}$ \\ University of Freiburg, Department of Psychology \\ Submitted June 2019
}

${ }^{1}$ My official name was "Sari Alsalti" at the time this bachelor thesis was submitted.
${ }^{2}$ My direct supervisor was Dr. Andre Aßfalg 


\begin{abstract}
Concern has been mounting over the reproducibility of findings in psychology and other empirical sciences. Large scale replication attempts found worrying results. The high rate of false findings in the published research has been partly attributed to scientists' engagement in questionable research practices (QRPs). I discuss reasons and solutions for this problem. Employing a content analysis of empirical studies published in the years 2007 and 2017, I found a decrease in the prevalence of QRPs in the investigated decade. I subsequently discuss possible explanations for the improvement as well as further potential contributors to the high rate of false findings in science. Most scientists agree that a change towards more open and transparent scientific practice on part of both scientists and publishers is necessary. Debate exists as to how this should be achieved. Keywords: Questionable Research Practices. Publication bias. Replication crisis.
\end{abstract}




\section{Contents}

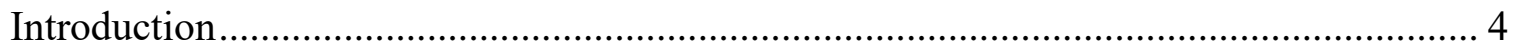

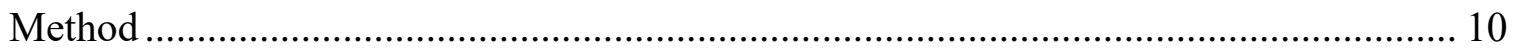

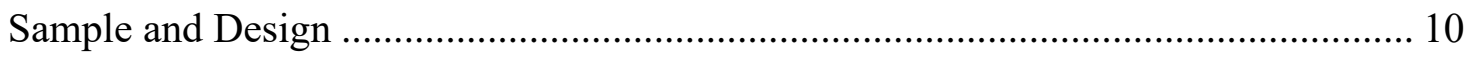

Indicator Variables and Coding Procedures ...................................................... 10

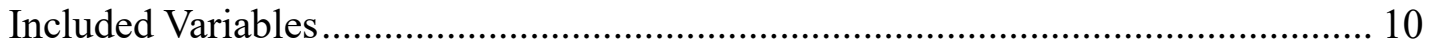

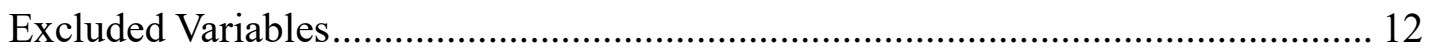

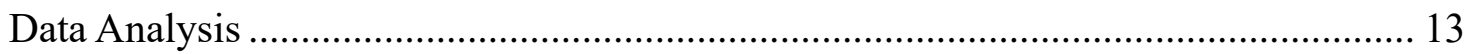

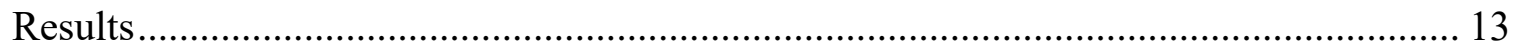

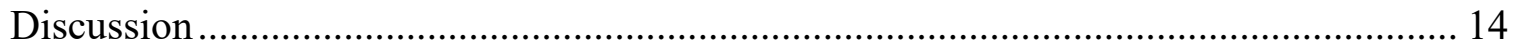




\section{Introduction}

There has been growing attention surrounding the "replication crisis" in psychology and the empirical sciences in general (Maxwell, Lau, \& Howard, 2015). This term describes the frequent failure to replicate results of previous studies. More scientists are searching for the underlying causes of this crisis and devising measures to deal with its effects. One of the most important contributors to this degradation of research quality is thought to be the several questionable research practices (QRPs) scientists systematically engage in (Bakker, van Dijk, \& Wicherts, 2012; Franco, Malhotra, \& Simonovits, 2016; Ioannidis, 2005; Simmons, Nelson, \& Simonsohn, 2011; Wicherts et al., 2016). QRPs, sometimes affectionately referred to as 'sloppy science'3 , are seen as the middle point between responsible conduct of research, which "[...] represents the ideal standard institutions and individuals endeavour to meet" (Steneck, 2006, p. 54), and outright misconduct that everyone agrees should be avoided, such as data fabrication and plagiarism. However, although previously not thought to be serious enough to warrant action (Steneck, 2006), much less frowned upon and more prevalent practices like flexibility in data collection, analysis and reporting are now considered likely to be far more detrimental to the quality of published research than stark misconduct (Ioannidis, 2005; John, Loewenstein, \& Prelec, 2012; Simmons et al., 2011). Martinson, Anderson, and de Vries (2005, p. 737) concluded in their survey study: “[...] our evidence suggests that mundane 'regular' misbehaviours present greater threats to the scientific enterprise than those caused by high-profile misconduct cases such as fraud."

The goal of this work was to determine whether differences in the relative importance of a journal (measured by its impact factor, IF) and in time of publication (years 2007 and 2017) are associated with differences in the quality of scientific practice. In other words, this work aims to answer the following research questions:

1. Is the prevalence of QRP indicators in empirical studies determined by the "prestige" of the journals the studies are published in?

${ }^{3}$ Although "sloppy science" and "QRPs" are sometimes used interchangeably. I choose to distinguish between them on account of their different semantic connotations. For example, I would rather describe omitting ES's as sloppy than questionable. On the other hand, HARKing (hypothesising after the results are known) is more questionable than sloppy to me. I do however mostly use "bias" and "QRPs" interchangeably, especially when referring to researchers' behaviour. 
2. Has the prevalence of QRP indicators in empirical studies changed between the years 2007 and $2017 ?$

In 2011, Simmons et al. reported an experiment which came to the result that listening to a certain song significantly reduces participants' age. Understandably, the authors did not try to replicate this "revolutionary" finding because that was not the goal of their study. Rather, they wanted to demonstrate with help of actual experiments and computer simulations how easy it was to acquire and report significant evidence for any hypothesis when exploiting enough of what the authors called researcher degrees of freedom (RDF). RDF represent the typical decisions researchers face when planning, collecting data, and analysing results (e.g. decisions about sample size, which variables should be included in the analysis etc.). For example, they obtained the significant age reduction result by collecting several variables, conducting several analyses on them and eventually choosing the combination (of dependent variable and covariate) that turned out to be significant. Furthermore, they flexibly recruited participants until this result reached the significance level. Finally, they subsequently only reported the significant results and failed to mention the rest of the collected variables (they enlightened the reader about their procedure in a later section of the article). According to the authors, this reporting behaviour "[...] fully adheres to currently acceptable reporting standards and is, not coincidentally, deceptively persuasive." (Simmons et al., 2011, p. 6). This sort of opportunistic exploitation of RDFs in collecting and analysing data with the goal of bringing the $p$-value below the predefined threshold for significance is also called $p$ hacking, which is a type of QRPs. (c.f. Head, Holman, Lanfear, Kahn, \& Jennions, 2015; Wicherts et al., 2016).

With the aid of computer simulations, Simmons et al. (2011) also calculated the likelihood of obtaining a false-positive result while exploiting different RDFs, for example, addition of 10 more observations per cell: $7.7 \%$ at the $p<.05$ level; addition of an independent variable: 9.5\%; combining both: $14.4 \%$. These results indicate that the almost universally assumed false-positive probability of $<.05$ is in most cases far from being accurate. Ioannidis (2005) similarly asserted that the probability that a research finding is false increases with smaller sample and effect sizes, greater number and lesser preselection of tested relationships as well as greater flexibility in designing studies and analysing their results. According to the author, these conditions create many biases that distort the supposed findings. 
Reports by survey studies assessing the prevalence of QRPs have yielded alarming results. A meta-analysis of 18 surveys (Fanelli, 2009) found that up to $33.7 \%$ of the surveyed researchers admitted having engaged in QRPs. Admission rates about the behaviour of colleagues were up to $72 \%$. Even higher numbers were reported by John et al. (2012). They asked research psychologists about their engagement in such QRPs as failing to report all dependent variables, collecting more data after seeing whether results were significant and failing to report all conditions, among others. Self-admission rates for these three practices were $78 \%, 72 \%$ and $42 \%$, respectively. All in all, 94\% of respondents admitted having engaged in at least one QRP. Furthermore, a systematic review of studies that gathered data from observations, sensitivity analyses and surveys found that 91\% of the 64 analysed studies found evidence for QRPs (Banks, Rogelberg, Woznyj, Landis, \& Rupp, 2016).

Hence, when considering both the gravity of QRPs' effects on Type I error rate and their high prevalence it becomes less difficult to make sense of the inflated proportion of irreplicable studies in published papers. Ioannidis (2005) went even so far as to proclaim the majority of published research false. Although this claim has been contested (e.g. Goodman \& Greenland, 2007a, 2007b; Jager \& Leek, 2014), large scale replication attempts (Begley \& Ellis, 2012; Open Science Collaboration, 2015) seem to support Ioannidis's claim; the Open Science Collaboration managed to only replicate 36 significant results out of the attempted 100 in psychology; Begley and Ellis succeeded in replicating 6 out of 53 significant results in haematology and oncology.

As to the reason researchers resort to QRPs, fingers mostly point to publication bias (Bakker et al., 2012; Easterbrook, Gopalan, Berlin, \& Matthews, 1991; Fanelli, 2010; Ioannidis, 2005; Sackett, 1979; Wicherts et al., 2016). Publication bias refers to the superior likelihood for results to be submitted by authors, and get published in journals, when they're significant in the hypothesised direction (Sackett, 1979). Knowledge of this bias (journals' preference for positive results), coupled with the pressure on scientists to incessantly and rapidly publish academic work in order to maintain or further their careers (Neill, 2008), drive them to do anything to produce publishable papers (Fanelli, 2010). This could include exploiting their RDF to sway their data to tell them what they want to be true (Head et al., 2015), claiming that a significant result of a secondary exploratory analysis (with no relation to the hypotheses) answers a main research question of their study (HARKing, Hypothesising after the results are known, Kerr, 1998) or in some rarer cases, outright fraud and data fabrication (al-Marzouki, Evans, Marshall, \& Roberts, 2005; 
Simonsohn, 2013). The other aspect of publication bias, also known as the file drawer problem, describes authors' tendency to suppress studies that do not go their way (i.e. not submit them for publication, but put them away in a file drawer, Franco, Malhotra, \& Simonovits, 2014; Rosenthal, 1979). Indeed, reporting only the results that support one's theory or position is a QRP in and of itself. It leads, as does publication bias on the parts of reviewers and editors, to an overrepresentation of positive results in the published literature (cf. reporting bias, Dickersin \& Min, 1993; Ioannidis \& Trikalinos, 2007; Pigott, Valentine, Polanin, Williams, \& Canada, 2013; Rosenthal, 1979). Sterling (1959) discussed publication bias and lamented its impact on published science over 50 years ago, maintaining it leads to an inflation of type I errors in the literature. This occurs due to scientists publishing significant results which were previously found null by other researchers and consequently remained unpublished. Beyond these reasons, other authors argue that scientists are not invulnerable to confirmation bias (Nickerson, 1998; Nuzzo, 2015), having the tendency to consciously and unconsciously steer their studies in the direction that would lead to an outcome which reaffirms their hypotheses. Hence, it can be argued that the existence of RDF in the first place is facilitates their exploitation to satisfy researcher's near constant motivation to confirm their presuppositions (Wicherts et al., 2016).

Since Ioannidis's (2005) and Simmons et al.’s (2011) impactful publications, several solutions to the problems regarding QRPs and the high false positive rate were proposed by scientists to reduce their effects. The most frequently advanced proposal is, to my knowledge, a preregistration system that requires authors to disclose all information pertaining to their study before commencing data collection (e.g. research questions, all dependent and independent variables investigated, how sample size is determined, whether subjects were excluded etc., Humphreys, Sanchez de la Sierra \& van der Windt, 2013; LeBel et al., 2013; Lilienfeld, 2017; van 't Veer \& GinerSorolla, 2016). In some cases, this submission (a "registered report") can be rejected or revised, corresponding to the typical journal review process. Registered reports can be granted an "in-principle acceptance" for publication before starting to collect data for the study. Consequently, this ensures that publication decisions on part of the journals are predominantly based on the quality of research questions and methods and not on the outcome (Hardwicke \& Ioannidis, 2018). The main aim of requiring authors to preregister their studies is to prevent them from HARKing, $p$ hacking and underreporting (Franco et al., 2016; Head et al., 2015; Kerr, 1998), thus rendering easier the distinction between confirmatory and exploratory analyses and results (Jonas \& Cesario, 
2016). Other recommendations include calls for the use of Bayesian statistics, posting materials and data pertaining to the published study, correcting alpha levels, and setting stricter a priori alpha levels (e.g. 0.001, Francis, 2012; Johnson, 2013; Johnson, Payne, Wang, Asher, \& Mandal, 2017; Simmons et al., 2011; Simonsohn, 2013). Pleas for solutions appear to have been heard, as there are now services that offer preregistration for all disciplines, with some journals even requiring it for publishing (Nosek \& Lakens, 2014).

Besides the proposals to battle the causes of QRPs, statisticians have also developed methods to detect their effects on published research. These methods are also used to assess the prevalence of QRPs without having to rely on survey data. Survey responses regarding delicate topics (such as involvement in potentially immoral or even illegal activities) are likely to be biased (Fanelli, 2009; John et al., 2012). Thus, detection methods that are independent of biased selfreports should offer incrementally valuable information regarding the prevalence of QRPs in the published literature (Matthes et al., 2015). So called 'bias tests' have been in use for over 2 decades (Ioannidis, Munafò, Fusar-Poli, Nosek, \& David, 2014). Most of these tests were developed to be applied to evaluate bias across several studies investigating the same topic. One such test, developed by Ioannidis and Trikalinos (2007), is applied to assess whether the number of statistically significant results in a set of studies is higher than what would be expected considering the effect sizes of the studies (for a review of bias tests, see Ioannidis et al., 2014). Bias tests applied to larger groups of studies cannot identify which single studies are affected more by bias, nor whether the authors or the journals were the source of the bias. Their main goal is rather to give insight into the average bias in a certain field. Measuring bias in single studies is considerably more difficult, as they do not offer as much data. Despite this, a few options for applying bias tests to single studies exist. For instance, bias can be detected by comparing studies' eventually published reports with their preregistration or protocol submissions, provided such submissions are available (Dwan, Gamble, Williamson, \& Kirkham, 2013). Furthermore, although conceptualized by Ioannidis and Trikalinos (2007) for meta-analyses, Francis $(2012,2014)$ successfully employed a variation of their test to detect an excess of significant results in single studies with multiple experiments (at least 4). 
Another approach was introduced by Matthes et al. (2015) experimental studies from the field of communication research, looking for indicators of QRP(e.g. lack of a priori power analysis, small sample sizes, case deletion etc.), which they compiled in a codebook. Loosely following Matthes et al.'s procedure (2015), I performed for the purpose of this study a content analysis of 40 cognitive psychological empirical studies. I accordingly also compiled the QRP indicators I examined in a codebook (see Table 1). However, my approach differed from that of Matthes et al. (2015) in two main aspects: 1. I pooled the individual indicator-variables into a global "dodginess score" (DS) by coding them in the same direction (e.g. no a priori power analysis $=1$, cases deleted $=1$, effect sizes reported $=0$ ) and summing them up. Since all variables were negatively coded as in the examples above, a greater DS meant 'sloppier' science, that is to say higher indication of QRPs. 2. Matthes et al. (2015) only investigated the influence of time on the prevalence of QRP indicators. Their findings were inconclusive (e.g. effect sizes were reported more frequently over time but the ratio of confirmed to falsified hypotheses increased). Besides time (the years 2007 and 2017), I also included the journal impact factor as an independent variable and explored the potential association of both variables with the DS. This was my reasoning regarding this decision: one could tentatively argue that more prestigious journals would tend to set stricter requirements for publication and accordingly accepts papers of higher quality. The 'prestige' of journals is usually represented by their impacts factors (IF). The journal IF refers to the yearly average number of citations to the scholarly articles published in that journal (McVeigh \& Mann, 2009). They are often employed as indirect measures of the relative importance of journals (Hoeffel, 1998). Following this, I expected journals with greater impact factors to publish papers characterised by better research practices (smaller DS). Furthermore, in light of the substantial developments in the academic field discussed above, a change on the part of scientists could also be expected. For example, even if not obligated, scientists might opt for more rigorous work now that they're more aware of the negative consequences of QRPs. Based on these considerations, I hypothesised an improvement in research practice between the years 2007 and 2017.

${ }^{4}$ I chose to adopt Matthes et al.'s approach because it was easier to implement in the limited I had to finish this work. Although bias tests could arguably produce more meaningful and informative results, the requirements for their use cannot be realistically met within the scope of this work. 


\section{Method}

\section{Sample and Design}

I obtained the 40 studies from the first 2007 and 2017 issues (January, February and March) of the journals Cognition and Memory \& Cognition . This yielded 10 data points for each combination of the manipulated variables: C07 (Cognition in 2007), C17 (C in 2017), MC07 (Memory * Cognition in 2007), MC17 (MC in 2017). The inclusion criteria for choosing the journals were: A. They differed in their IFs and B. They published only empirical studies in the field of cognitive psychology. The second criterion turned out to be not fully met as 3 non-empirical studies were found and replaced by studies from later issues. The mean IFs of the journals $\mathrm{C}$ and $\mathrm{MC}$ aggregated over the years 2007 and 2017 were 4.13 (C) and 1.6 (MC) (ResearchGate, n.d.-a, n.d.-b).

\section{Indicator Variables and Coding Procedures}

I collected 14 variables from the chosen studies. Not all variables were eventually included in the analysis and only 8 indicator variables were used to create the DS (see Data Analysis). In studies that reported more than one experiment, each experiment was examined separately. Data from the individual experiments were subsequently aggregated to their respective studies.

\section{Included Variables}

\section{A priori power analysis}

Coding: authors reported having performed an a priori power analyses $\rightarrow 0$, no a priori power analysis reported $\rightarrow 1$.

Explanation: Determining the sample size before the start of data collection partially restraints the researcher's potential temptation to $p$-hack, e.g. collect data until an effect becomes significant (Simmons et al., 2011). Determining the sample size using an a priori power analysis has the additional advantage of collecting just as many data points as necessary (Cohen, 1990).

\section{Smallest $n$}

Coding: the sample size of the smallest subgroup. When not reported: $N$ (total sample size)/ number of subgroups. $n<20 \rightarrow 2,20 \leq n<40 \rightarrow 1, n \geq 40 \rightarrow 0$, calculation of $n$ not possible (e.g. because no sample at all is reported) $\rightarrow 2$

Explanation: designs with $n$ 's smaller than 20 can seldom possess enough power to detect an effect (Simmons et al., 2011). 


\section{Balanced design}

Coding: $n$ 's are not equal $\rightarrow 1, n$ 's are equal $\rightarrow 0$, not applicable (NA, e.g. within-subjects design) $\rightarrow 0$.

Explanation: Most studies I examined employed ANOVAs to test their hypotheses. ANOVAs with balanced designs yield more precise more precise parameter estimates (e.g. sums of squares of factors are independent of each other) that facilitate straightforward interpretation of the results (Shaw \& Mitchell-Olds, 1993). Shaw and Mitchell-Olds (1993, p. 1640) summarised the problems with unbalanced designs thus, "[...] loss of balance causes ambiguities that plague the processes of estimating the parameters, partitioning the sums of squares and testing the hypotheses of interest". Measures should be taken during the analyses to correct for unequal sample sizes.

\section{Confidence intervals (CIs)}

Coding: no CIs reported $\rightarrow 2$, at least one CI reported $\rightarrow 1$, CIs reported for all results $\rightarrow$ 0

Explanation: CIs not only provide all the information a significance test can, but also offer an estimation of the uncertainty of one's estimated parameters. Statisticians have advocated for their use for decades (Cohen, 1994).

\section{Effect sizes (ESs)}

Coding: no ESs reported $\rightarrow 2$, at least one ES reported $\rightarrow 1$, ESs reported for all results $\rightarrow 0$

Explanation: Frank Yates, one of the pioneers of $20^{\text {th }}$ century statistics, complained about exaggerated attention to significance as early as 1951, saying " [...] it has caused scientific research workers to pay undue attention to the results of the tests of significance they perform on their data, particularly data derived from experiments, and too little to the estimates of the magnitude of the effects they are investigating. "ESs, along with CIs, are essential for interpreting results and are certainly more informative than $p$-values (Cohen, 1994; Funder et al., 2014; Nosek \& Lakens, 2014).

\section{Data points exclusion}

Coding: at least one data point excluded after/during the analysis (e.g. due to outlier) $\rightarrow 1$, no data points excluded (or data points excluded before the data analysis, e.g. due to incompliance with instructions during the experiment) $\rightarrow 0$ 
Explanation: this variable is dependent on the variable 'results without data point exclusion' (see below).

\section{Raw data}

Coding: authors made their raw data available online $\rightarrow 0$, raw data not available $\rightarrow 1$

Explanation: results are much less likely to be fabricated if the raw data are available publicly where others can verify them (Simonsohn, 2013). Posting raw data online also makes it easier to prevent and detect reporting and analysis errors (Wicherts \& Bakker, 2012).

\section{Confirmation vs. Falsification}

Coding: at least one result was reported to have been unexpected or one hypothesis was reported to be not confirmed $\rightarrow 0$, all hypotheses confirmed $\rightarrow 1$

Explanation: a complete absence of null results indicates bias, such as HARKing or publication bias (Matthes et al., 2015).

\section{Excluded Variables}

\section{Total sample size $N$}

Subgroup sample sizes are more informative than total sample sizes, as they already account for the number of independent variable manipulations. Including total sample size in the analysis was therefore not necessary.

\section{P-values}

Following Matthes et al. (2015), I wanted to investigate the prevalence of $p$-values slightly under the significant level, as they give a hint of $p$-hacking (Masicampo \& Lalande, 2012). However, coding collected $p$-values proved to be difficult. $P$-values were very frequently reported as ranges (e.g. $p<.001$ ) and when exact $p$-values were given, they were very rarely close to the significance level. Furthermore, there was an immense variability in the number of $p$-values reported, which made selecting a sample of them without bias on my part difficult.

\section{Alpha correction}

Correction of $\alpha$-levels in accordance to number of conducted analyses reduces the probability of Type I errors (Glickman, Rao, \& Schultz, 2014). This variable was excluded due to mistakes in collecting its data. I incorrectly coded this variable negatively when more than one $p$-value was reported in one analysis without correcting the alpha levels (e.g. 2x2 ANOVA). 


\section{Covariates}

This variable is also dependent on the 'results without data point exclusion'. No covariates were employed in the 40 studies I analysed.

\section{Results without covariate(s)/Data point exclusion(s)}

When data points are excluded or covariates are included in the design, it is crucial that results before these changes be reported. Otherwise, it's impossible to know the extent to which the results rely on these changes (Simmons et al., 2011).

\section{Data Analysis}

The global dodginess score (DS) was constructed by summing up the 8 included indicator variables, which were all coded negatively (e.g. no raw data reported $\rightarrow 1$ ). A higher DS of a study therefore signifies a higher prevalence of QRP indicators. Data from the individual experiments were previously aggregated to their respective studies.

A 2x2 between-studies ANOVA on the DSs with the factors year (2007 and 2017) and journal (C and $\mathrm{MC}$ ) was conducted. Furthermore, I computed Bayes factors (BFs) for the main effects and the interaction effect using JASP 0.9.2.0 (jasp-stats.org). I employed Shapiro-WilkTests to test for normal distribution in the 4 subgroups. Histograms and Q-Q plots were checked as well. A Levene's test was also utilised to test for homogeneity of variances. Significance levels were set to 5\%. The data analysis was performed using SPSS 25 (ibm.com/analytics/spss-statisticssoftware).

\section{Results}

The Shapiro-Wilk-Tests conducted on the 4 subgroups gave no indication of a significant deviation of the normal distribution assumption (all $p$-values $>.31, \alpha=.05$ ). While the histograms didn't show a normal distribution (which is to be expected with $n=10$ ), Q-Q plots supported the conclusion of near normal distribution. The Levene's test indicated that the data were unlikely under assumption of variance homogeneity. Due to these results, I couldn't say for sure that the assumptions for ANOVA were met, which is why I conducted an additional nonparametric test to validate the ANOVA's main result (see below.)

The mean DSs of the 4 subgroups in addition to their 95\% CIs are depicted in Figure 1. The 2x2 (year x journal) ANOVA showed that mean DSs were significantly higher in the year 2007 than $2017, F(1,36)=33.5, p<.001, \eta_{\mathrm{p}}^{2}=.483, B F_{10}=17827.04$. The BF conveys that there is 
17827 times more evidence for the model with year effect compared to the null model (the model including only the intercept but no effects). The evidence for main effect journal and the interaction effect was negligible. Main effect journal: $F(1,36)=0.121, p=.730, \eta_{\mathrm{p}}^{2}=.003, B F_{10}=0.31$. This $\mathrm{BF}$ indicates that there is about 3 times more evidence for the null model than the model with the main effect journal. Interaction effect: $F(1,36)<0.001, p=.990, \eta_{\mathrm{p}}^{2}<0.001$ Seeing that the assumptions of the ANOVA were not fully met, I performed a median test to validate the effect of the main effect year (2007 vs. 2017). The median test came to similar results, $\chi^{2}(1,40)=14.40, p$ $<.001$.

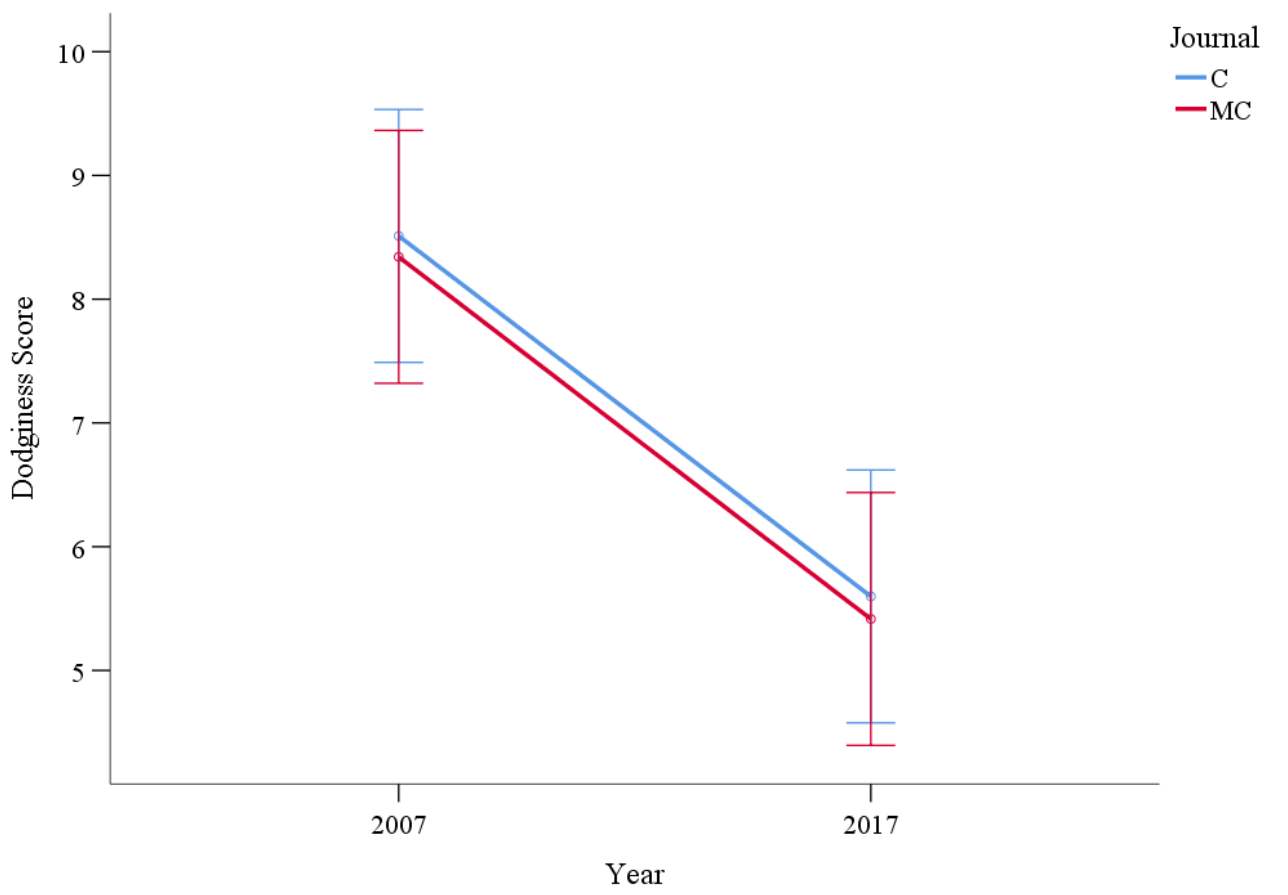

Figure 1: Mean global dodginess scores (DSs) of the 4 subgroups, Cognition (C) 2007, Cognition 2017, Memory \& Cognition (MC) 2007 and Memory \& Cognition 2017. Greater DS values imply higher questionable research practices prevalence. The error bars represent $95 \%$ confidence intervals.

\section{Discussion}

The main aim of this work was to investigate the influence of time and the prestige of the journal on the quality of published studies. To do this, I measured the variation in the prevalence of QRP indicators in studies published by two journals with different impact factors (Cognition and Memory \& Cognition) in the years 2007 and 2017. I expected the prevalence to be higher (greater DS) in the year 2007 and in the "less prestigious" journal. My data suggested a positive 
effect of time, as DSs decreased significantly between 2007 and 2017 independent of journal. The data did not support my hypothesis regarding journal prestige (represented by the IF). No interaction effect was expected nor was one found.

Ample caution is advised when interpreting these results, as there are several limitations (mostly due to time restrictions) to the methodological approach implemented in this work. First of all, the main advantage of content analyses (and bias tests) of QRPs over surveys is believed to be their independence of researchers' willingness to say the truth (Matthes et al., 2015). However, this claim is arguable at best, since this can be said only about a handful of QRP indicators. For instance, authors can easily falsely report having determined the sample size using an a priori power analysis or simply omit mentioning having excluded data points. Doubtless, there are clear signs of 'sloppy science', such as neglecting to report CIs or ESs. It remains nevertheless extremely difficult to detect actual QRPs such as $p$-hacking as long as the authors know how to conceal them. Secondly, I faced considerable difficulties during both the data collection and the coding procedures. For example, I had originally intended to only consider ESs, CIs and $p$-values reported in the results of the focal analyses (i.e. analyses with the aim of answering main research questions). This proved to be a near impossible task to achieve in the time allocated for this work, as hypotheses or research questions were very rarely explicitly communicated. Rather, it would have been necessary to read and understand the entire paper to identify its specific research aims and competently associate them with the results. Naturally, this problem affected examination of the variable "confirmation vs. falsification" the most. Relatedly, the data collection and coding procedures were all the less reliable for not having been validated by another rater. It is common in cases where measurements are subject to error or bias to have several raters asses the interrater reliability, as was done by Matthes et al. (2015). Thirdly, I coded most variables uniformly as " 1 " to indicate the negative outcome. This procedure is suboptimal at best, as it is doubtful whether the same weight of "questionable practice" should be assigned to all indicators (e.g. balances design vs. a priori power analysis). Finally, it goes without saying that future studies with similar research questions should, besides considering the above-mentioned aspects, employ larger sample sizes. This is indispensable for reaching reliable and valid results.

Despite these limitations, the large effect of time I found is not implausible. This apparent improvement in research practices over time can best be explained by the developments the scientific publishing has experienced in the last decade as well as the rising awareness of QRPs. These 
developments probably emerged as a reaction to the stir caused by important publications written by concerned researchers (e.g. Ioannidis, 2005; John et al., 2012; Simmons et al., 2011). These papers showed the extent to which QRPs can compromise the quality of published research as well as how prevalent such practices were. The outcome of engaging in QRPs (and publication bias) is, according to numerous authors (e.g. Bakker et al., 2012; Ioannidis, 2005; Ioannidis \& Trikalinos, 2007; Open Science Collaboration, 2015; Rosenthal, 1979; Simmons et al., 2011; Sterling, 1959) an excessively disproportionate amount of publications with positive results that fail to replicate with an alarmingly high likelihood. Preregistration has been already extensively adopted by journals and scientific organisation to minimise the damage to future publications (Humphreys et al., 2013; LeBel et al., 2013; Lilienfeld, 2017). Despite challenges to its success and issues in its implementation (Francis, 2012; Hardwicke \& Ioannidis, 2018), the preregistration system has already generated noteworthy results; analyses of preregistered studied have demonstrated an increase in the publication of null findings (Allen \& Mehler, 2019; Kaplan \& Irvin, 2015).

Regarding the complicated relationship between QRPs and research quality, I must emphasise that blaming scientists and their engagement in QRPs for everything wrong with science would be both inaccurate and unfair. Ioannidis (2005) named several other reasons for the high rate of false findings in published research, with null hypothesis significance testing (NHST) itself being one of the most important. The discussion over the advantages and disadvantages of the "Fisherian" method began very early in the days after it spread, initiated by another statistician (and colleague of Fisher), Yates. Yates (1951) admitted that, although the adoption of frequentist statistics developed by Fisher lead to an improvement in the "accuracy and certainty of experimental results" (p. 32), it also created a tendency by scientists to focus too much on whether the results are significant and too little on the size of these results. Small effects are more likely to have been found significant by chance than larger effects (Johnson et al., 2017). In his position paper, Antonakis (2017) argued that science is suffering from serious "diseases" that suppress its ability to produce useful and reliable finding. Significosis, as he dubbed the disease describing the immoderate focus on $p$-values below an arbitrary threshold, distorts the 'natural' ratio of significant to null results in published research, as significant results are much more likely to get published than null results (Gerber \& Malhotra, 2008). It is also worth mentioning that this arbitrary dichotomisation of results into significant or non-significant based on the $p$-value, a continuous measure, was not the intention of the founders of statistical inference (Sterne \& Smith, 2001). The 
interpretation of the $p$-value is, according to Fisher (1951), up to the researcher and should depend on the type of study and relevant evidence available (Sterne \& Smith, 2001). More evidence for the mediocrity of .05 NHST was provided by (Ioannidis, 2008). Using Bayesian analyses, he found that up to $77 \%$ of significant epidemiological correlations had less than strong support to their credibility.

Unfortunately, NHST's pitfalls don't stop there. More than 20 years ago, Cohen (1994) quoted Bakan as having said back in 1966 that it was not original to talk about the "mischief" associated with NHST and that "everyone" knew about it (p. 423). The fact that scientists (e.g. Colquhoun, 2014, 2017; Greenland et al., 2016; Hubbard, 2011) are still discussing the issue surrounding the alarmingly common misinterpretations of $p$-values goes to show how deeply rooted and persistent this problem is. One of the most frequent such misinterpretations is that $p$-values give the probability that Ho is true (Cohen, 1994). Cohen (1990) even reported researchers formulating their research hypotheses as null hypotheses and subsequently interpreting the resulting nonsignificant $p$-values as confirmation. Meehl summed up NHST as a "[...] a potent but sterile intellectual rake who leaves in his merry path a long train of ravished maidens but no viable scientific offspring" (1967, p. 265, as cited in Cohen, 1994). As one would expect, solutions to the problems of NHST have been offered. The proposals displayed 4 degrees of deviation from the relatively common practice of only reporting $p$-values and interpreting them as significant or not: 1 . Reporting CIs and ESs in addition to $p$-values. (e.g. Cohen, 1994; du Prel, Hommel, Röhrig, \& Blettner, 2009); 2. Decreasing alpha levels (e.g. Johnson, 2013; Johnson et al., 2017); 3. Reporting $p$-values and CIs but interpreting them as continuous measures, thus abolishing NHST (e.g. Amrhein, Greenland, \& McShane, 2019; Colquhoun, 2014, 2017; Sterne \& Smith, 2001; Wasserstein, Schirm, \& Lazar, 2019); 4. Using Bayesian statistics (Francis, 2012). All but the first proposal have been met with opposition (Simmons et al., 2011). Even Ioannidis, who on more than one occasion demonstrated the negative sides of NHST (e.g. Ioannidis, 2005, 2008), spoke against abandoning statistical significance, arguing that this "[...] would give bias a free pass" (Ioannidis, 2019a, p. 1, 2019b). The debate is still ongoing.

Another disease with which empirical science appears to be afflicted is neophilia (Antonakis, 2017). It describes another form of publication bias, an inordinate preference for novel results. Combine the bias for positive and novel findings with journals' very restricted capacity for publication and you get the conditions that force scientists to aim not for sound, reliable findings, 
but rather for findings that appear to support their hypotheses immaculately (Giner-Sorolla, 2012; Nosek, Spies, \& Motyl, 2012). Consequently, these poor publishing standards combined with the relentless pressure on scientists to produce publishable findings incentivise them to resort to QRPs (Bakker et al., 2012; Fanelli, 2009; Ioannidis, 2005; Simmons et al., 2011; Simonsohn, 2013; Wicherts et al., 2016), not even attempting to publish null findings for certainty of their non-existent prospects of being published (Franco et al., 2014, 2016) and crucially, neglect replication attempts (Antonakis, 2017; Giner-Sorolla, 2012; Nosek et al., 2012). Replication has been and is still considered by many as one of the most vital constituents of science (e.g. Cohen, 1994; Moonesinghe, Khoury, \& Janssens, 2007; Simmons et al., 2011; Simons, 2014). It is only by replicating its findings that science fulfils its requirement for "self-correction" (Ioannidis, 2012). There has been debate over which form replications should take; direct or conceptual replication; and if direct, by the same laboratories that produced the original findings or by others (Munafò \& Davey Smith, 2018; Nosek \& Lakens, 2014; Simmons et al., 2011; Simons, 2014; Stroebe \& Strack, 2014). However, no replication study in any shape or size has a viable chance of being deemed worthy of publishing by most journals (Makel, Plucker, \& Hegarty, 2012; Nosek et al., 2012). The obvious solution to this problem would be changing the current dysfunctional publication norms (Nosek et al., 2012). This will certainly not be easy to achieve but the introduction of preregistration is a step in the right direction (Nosek \& Lakens, 2014). There are indications that publication rate of replication studies has been increasing over the last decades (Makel et al., 2012).

However, there are those that would deem this discussion unwarranted as they don't believe in the urgency of these problems; Ioannidis's (2005) claims about the rate of false findings in the published research were not the only ones to be contested (Goodman \& Greenland, 2007a, 2007b; Jager \& Leek, 2014). There are those that deny the existence of the replication crisis or regard its extent as exaggerated (Maxwell et al., 2015; Stroebe \& Strack, 2014). Maxwell et al. (2015) questioned the findings by large scale replication attempts, arguing that most replication studies are underpowered. Doubts have also been raised about the high prevalence of QRPs reported by surveys. Fiedler and Schwarz (2016) claimed that John et al.'s (2012) findings, due to questionable interpretation of survey data, grossly overestimated the frequency of QRP's.

These might be legitimate points of criticism. Nonetheless, several lines of evidence have demonstrated that the empirical sciences, including psychology, are not doing well. The assumed false positive rate of $5 \%$ can by no means be corroborated in the published literature. Although the 
results presented in this work suggest an improvement in research practice, science has a long way to go to get rid of its ailments, including arigorium (a deficiency in rigour, Antonakis, 2017). In a “scientific utopia" (Nosek et al., 2012), most scientists would only engage in responsible research practice (Steneck, 2006) as incentives would be restructured to promote truth, not publishibility. Which concrete measures must be undertaken to reach this utopia is understandably a matter of discussion. However, any change has to involve a more open and tolerant publishing system along with a more informed and transparent methodological practices on the part of scientists. 


\section{References}

al-Marzouki, S., Evans, S., Marshall, T., \& Roberts, I. (2005). Are these data real? Statistical methods for the detection of data fabrication in clinical trials. BMJ, 331, 267. https://doi.org/10.1136/bmj.331.7511.267

Allen, C., \& Mehler, D. M. A. (2019). Open science challenges, benefits and tips in early career and beyond. PLOS Biology, 17, e3000246. https://doi.org/10.1371/journal.pbio.3000246

Amrhein, V., Greenland, S., \& McShane, B. (2019). Scientists rise up against statistical significance. Nature, 567, 305-307. https://doi.org/10.1038/d41586-019-00857-9

Antonakis, J. (2017). On doing better science: From thrill of discovery to policy implications. The Leadership Quarterly, 28, 5-21. https://doi.org/10.1016/J.LEAQUA.2017.01.006

Bakan, D. (1966). The test of significance in psychological research. Psychological Bulletin, 66, 423-437. https://doi.org/10.1037/h0020412

Bakker, M., van Dijk, A., \& Wicherts, J. M. (2012). The Rules of the Game Called Psychological Science. Perspectives on Psychological Science, 7, 543-554. https://doi.org/10.1177/1745691612459060

Banks, G. C., Rogelberg, S. G., Woznyj, H. M., Landis, R. S., \& Rupp, D. E. (2016, September 25). Editorial: Evidence on Questionable Research Practices: The Good, the Bad, and the Ugly. Journal of Business and Psychology, 31, 323-338. https://doi.org/10.1007/s10869016-9456-7

Begley, C. G., \& Ellis, L. M. (2012). Raise standards for preclinical cancer research. Nature, 483, 531-533. https://doi.org/10.1038/483531a

Cohen, J. (1990). Things I have learned. American Psychologist, 45, 1304-1312. https://doi.org/10.1037/0003-066X.45.12.1304

Cohen, J. (1994). The earth is round ( $<$.05): Rejoinder. American Psychologist, 50, 11031103. https://doi.org/10.1037/0003-066X.50.12.1103

Colquhoun, D. (2014). An investigation of the false discovery rate and the misinterpretation of $\mathrm{p}$ values. Royal Society Open Science, 1, 140216-140216. https://doi.org/10.1098/rsos.140216

Colquhoun, D. (2017). The reproducibility of research and the misinterpretation of p-values. Royal Society Open Science, 4, 171085. https://doi.org/10.1098/rsos.171085

Dickersin, K., \& Min, Y.-I. (1993). Publication Bias: The Problem That Won't Go Away. Annals 
of the New York Academy of Sciences, 703, 135-148. https://doi.org/10.1111/j.17496632.1993.tb26343.x

du Prel, J.-B., Hommel, G., Röhrig, B., \& Blettner, M. (2009). Confidence interval or p-value?: part 4 of a series on evaluation of scientific publications. Deutsches Ärzteblatt International, 106, 335-339. https://doi.org/10.3238/arztebl.2009.0335

Dwan, K., Gamble, C., Williamson, P. R., \& Kirkham, J. J. (2013). Systematic Review of the Empirical Evidence of Study Publication Bias and Outcome Reporting Bias - An Updated Review. PLoS ONE, 8, e66844. https://doi.org/10.1371/journal.pone.0066844

Easterbrook, P. ., Gopalan, R., Berlin, J. ., \& Matthews, D. . (1991). Publication bias in clinical research. The Lancet, 337, 867-872. https://doi.org/10.1016/0140-6736(91)90201-Y

Fanelli, D. (2009). How many scientists fabricate and falsify research? A systematic review and meta-analysis of survey data. PLoS ONE, 4, e5738. https://doi.org/10.1371/journal.pone.0005738

Fanelli, D. (2010). Do pressures to publish increase scientists' bias? An empirical support from US states data. PLoS ONE, 5, e10271. https://doi.org/10.1371/journal.pone.0010271

Fiedler, K., \& Schwarz, N. (2016). Questionable Research Practices Revisited. Social Psychological and Personality Science, 7, 45-52. https://doi.org/10.1177/1948550615612150

Fisher, R. A. (1951). Statistical methods for research workers (2nd ed.). Edinburgh: Oliver \& Boyd.

Francis, G. (2012). Too good to be true: Publication bias in two prominent studies from experimental psychology. Psychonomic Bulletin \& Review, 19, 151-156. https://doi.org/10.3758/s13423-012-0227-9

Francis, G. (2014, October 18). The frequency of excess success for articles in Psychological Science. Psychonomic Bulletin and Review. https://doi.org/10.3758/s13423-014-0601-x

Franco, A., Malhotra, N., \& Simonovits, G. (2014). Social science. Publication bias in the social sciences: unlocking the file drawer. Science (New York, N.Y.), 345, 1502-1505. https://doi.org/10.1126/science. 1255484

Franco, A., Malhotra, N., \& Simonovits, G. (2016). Underreporting in Psychology Experiments. Social Psychological and Personality Science, 7, 8-12. https://doi.org/10.1177/1948550615598377 
Funder, D. C., Levine, J. M., Mackie, D. M., Morf, C. C., Sansone, C., Vazire, S., \& West, S. G. (2014). Improving the Dependability of Research in Personality and Social Psychology. Personality and Social Psychology Review, 18, 3-12. https://doi.org/10.1177/1088868313507536

Gerber, A. S., \& Malhotra, N. (2008). Publication Bias in Empirical Sociological Research. Sociological Methods \& Research, 37, 3-30. https://doi.org/10.1177/0049124108318973

Giner-Sorolla, R. (2012). Science or Art? How Aesthetic Standards Grease the Way Through the Publication Bottleneck but Undermine Science. Perspectives on Psychological Science, 7, 562-571. https://doi.org/10.1177/1745691612457576

Glickman, M. E., Rao, S. R., \& Schultz, M. R. (2014). False discovery rate control is a recommended alternative to Bonferroni-type adjustments in health studies. Journal of Clinical Epidemiology, 67, 850-857. https://doi.org/10.1016/J.JCLINEPI.2014.03.012

Goodman, S., \& Greenland, S. (2007a). Assessing the Unreliability of the Medical Literature. Johns Hopkins University, Dept. of Biostatistics Working Papers. Retrieved from https://biostats.bepress.com/jhubiostat/paper135

Goodman, S., \& Greenland, S. (2007b). Why Most Published Research Findings Are False: Problems in the Analysis. PLoS Medicine, 4, e168. https://doi.org/10.1371/journal.pmed.0040168

Greenland, S., Senn, S. J., Rothman, K. J., Carlin, J. B., Poole, C., Goodman, S. N., \& Altman, D. G. (2016). Statistical tests, $P$ values, confidence intervals, and power: a guide to misinterpretations. European Journal of Epidemiology, 31, 337-350. https://doi.org/10.1007/s10654-016-0149-3

Hardwicke, T. E., \& Ioannidis, J. P. A. (2018). Mapping the universe of registered reports. Nature Human Behaviour. BITSS. https://doi.org/10.1038/s41562-018-0444-y

Head, M. L., Holman, L., Lanfear, R., Kahn, A. T., \& Jennions, M. D. (2015). The Extent and Consequences of P-Hacking in Science. PLOS Biology, 13, e1002106. https://doi.org/10.1371/journal.pbio.1002106

Hoeffel, C. (1998, December 1). Journal Impact Factors. Allergy: European Journal of Allergy and Clinical Immunology. John Wiley \& Sons, Ltd (10.1111). https://doi.org/10.1111/j.1398-9995.1998.tb03848.x

Hubbard, R. (2011). The widespread misinterpretation of $p$-values as error probabilities. Journal 
of Applied Statistics, 38, 2617-2626. https://doi.org/10.1080/02664763.2011.567245

Humphreys, M., Sanchez de la Sierra, R., \& van der Windt, P. (2013). Fishing, Commitment, and Communication: A Proposal for Comprehensive Nonbinding Research Registration. Political Analysis, 21, 1-20. https://doi.org/10.1093/pan/mps021

Ioannidis, J. P. A. (2005). Why Most Published Research Findings Are False. PLoS Medicine, 2, e124. https://doi.org/10.1371/journal.pmed.0020124

Ioannidis, J. P. A. (2008). Effect of formal statistical significance on the credibility of observational Associations. American Journal of Epidemiology, 168, 374-383. https://doi.org/10.1093/aje/kwn156

Ioannidis, J. P. A. (2012). Why Science Is Not Necessarily Self-Correcting. Perspectives on Psychological Science, 7, 645-654. https://doi.org/10.1177/1745691612464056

Ioannidis, J. P. A. (2019a). Retiring statistical significance would give bias a free pass. Nature, 567, 461-461. https://doi.org/10.1038/d41586-019-00969-2

Ioannidis, J. P. A. (2019b). The Importance of Predefined Rules and Prespecified Statistical Analyses. JAMA, 321, 2067. https://doi.org/10.1001/jama.2019.4582

Ioannidis, J. P. A., Munafò, M. R., Fusar-Poli, P., Nosek, B. A., \& David, S. P. (2014).

Publication and other reporting biases in cognitive sciences: detection, prevalence, and prevention. Trends in Cognitive Sciences, 18, 235-241.

https://doi.org/10.1016/J.TICS.2014.02.010

Ioannidis, J. P. A., \& Trikalinos, T. A. (2007). An exploratory test for an excess of significant findings. Clinical Trials, 4, 245-253. https://doi.org/10.1177/1740774507079441

Jager, L. R., \& Leek, J. T. (2014). An estimate of the science-wise false discovery rate and application to the top medical literature. Biostatistics, 15, 1-12.

https://doi.org/10.1093/biostatistics/kxt007

John, L. K., Loewenstein, G., \& Prelec, D. (2012). Measuring the Prevalence of Questionable Research Practices With Incentives for Truth Telling. Psychological Science, 23, 524-532. https://doi.org/10.1177/0956797611430953

Johnson, V. E. (2013). Revised standards for statistical evidence. Proceedings of the National Academy of Sciences of the United States of America, 110, 19313-19317. https://doi.org/10.1073/pnas.1313476110

Johnson, V. E., Payne, R. D., Wang, T., Asher, A., \& Mandal, S. (2017). On the Reproducibility 
of Psychological Science. Journal of the American Statistical Association, 112, 1-10. https://doi.org/10.1080/01621459.2016.1240079

Jonas, K. J., \& Cesario, J. (2016, September 24). How can preregistration contribute to research in our field? Comprehensive Results in Social Psychology, 1, 1-7. https://doi.org/10.1080/23743603.2015.1070611

Kaplan, R. M., \& Irvin, V. L. (2015). Likelihood of Null Effects of Large NHLBI Clinical Trials Has Increased over Time. PLOS ONE, 10, e 0132382. https://doi.org/10.1371/journal.pone.0132382

Kerr, N. L. (1998). HARKing: Hypothesizing After the Results are Known. Personality and Social Psychology Review, 2, 196-217. https://doi.org/10.1207/s15327957pspr0203_4

LeBel, E. P., Borsboom, D., Giner-Sorolla, R., Hasselman, F., Peters, K. R., Ratliff, K. A., \& Smith, C. T. (2013). PsychDisclosure.org. Perspectives on Psychological Science, 8, 424432. https://doi.org/10.1177/1745691613491437

Lilienfeld, S. O. (2017). Psychology's Replication Crisis and the Grant Culture: Righting the Ship. Perspectives on Psychological Science, 12, 660-664. https://doi.org/10.1177/1745691616687745

Makel, M. C., Plucker, J. A., \& Hegarty, B. (2012). Replications in Psychology Research: How Often Do They Really Occur? Perspectives on Psychological Science, 7, 537-542. https://doi.org/10.1177/1745691612460688

Martinson, B. C., Anderson, M. S., \& de Vries, R. (2005). Scientists behaving badly. Nature, 435, 737-738. https://doi.org/10.1038/435737a

Masicampo, E. J., \& Lalande, D. R. (2012). A peculiar prevalence of p values just below .05. Quarterly Journal of Experimental Psychology, 65, 2271-2279. https://doi.org/10.1080/17470218.2012.711335

Matthes, J., Marquart, F., Naderer, B., Arendt, F., Schmuck, D., \& Adam, K. (2015). Questionable Research Practices in Experimental Communication Research: A Systematic Analysis From 1980 to 2013. Communication Methods and Measures, 9, 193-207. https://doi.org/10.1080/19312458.2015.1096334

Maxwell, S. E., Lau, M. Y., \& Howard, G. S. (2015). Is psychology suffering from a replication crisis?: What does "failure to replicate" really mean? American Psychologist, 70, 487-498. https://doi.org/10.1037/a0039400 
Moonesinghe, R., Khoury, M. J., \& Janssens, A. C. J. W. (2007). Most Published Research Findings Are False_-But a Little Replication Goes a Long Way. PLoS Medicine, 4, e28. https://doi.org/10.1371/journal.pmed.0040028

Munafò, M. R., \& Davey Smith, G. (2018). Robust research needs many lines of evidence. Nature, 553, 399-401. https://doi.org/10.1038/d41586-018-01023-3

Nickerson, R. S. (1998). Confirmation bias: A ubiquitous phenomenon in many guises. Review of General Psychology, 2, 175-220. https://doi.org/10.1037/1089-2680.2.2.175

Nosek, B. A., \& Lakens, D. (2014, May 1). Registered reports: A method to increase the credibility of published results. Social Psychology. Hogrefe Publishing. https://doi.org/10.1027/1864-9335/a000192

Nosek, B. A., Spies, J. R., \& Motyl, M. (2012). Scientific Utopia: II. Restructuring Incentives and Practices to Promote Truth Over Publishability. Perspectives on Psychological Science, 7, 615-631. https://doi.org/10.1177/1745691612459058

Nuzzo, R. (2015). How scientists fool themselves - and how they can stop. Nature, 526, 182185. https://doi.org/10.1038/526182a

Open Science Collaboration. (2015). Estimating the reproducibility of psychological science. Science, 349, aac4716. https://doi.org/10.1126/SCIENCE.AAC4716

Pigott, T. D., Valentine, J. C., Polanin, J. R., Williams, R. T., \& Canada, D. D. (2013). OutcomeReporting Bias in Education Research. Educational Researcher, 42, 424-432. https://doi.org/10.3102/0013189X13507104

ResearchGate. (n.d.-a). No Title. Retrieved from https://www.researchgate.net/journal/0090502X_Memory_Cognition

ResearchGate. (n.d.-b). No Title. Retrieved from https://www.researchgate.net/journal/00100277_Cognition

Rosenthal, R. (1979). The file drawer problem and tolerance for null results. Psychological Bulletin, 86, 638-641. https://doi.org/10.1037/0033-2909.86.3.638

Sackett, D. L. (1979). Bias in analytic research. Journal of Chronic Diseases, 32, 51-63. https://doi.org/10.1016/0021-9681(79)90012-2

Shaw, R. G., \& Mitchell-Olds, T. (1993). Anova for Unbalanced Data: An Overview. Ecology, 74, 1638-1645. https://doi.org/10.2307/1939922

Simmons, J. P., Nelson, L. D., \& Simonsohn, U. (2011). False-positive psychology: Undisclosed 
flexibility in data collection and analysis allows presenting anything as significant. Psychological Science, 22, 1359-1366. https://doi.org/10.1177/0956797611417632

Simons, D. J. (2014). The Value of Direct Replication. Perspectives on Psychological Science, 9 , 76-80. https://doi.org/10.1177/1745691613514755

Simonsohn, U. (2013). Just Post It: The Lesson From Two Cases of Fabricated Data Detected by Statistics Alone. Psychological Science, 24, 1875-1888. https://doi.org/10.1177/0956797613480366

Steneck, N. H. (2006). Fostering integrity in research: Definitions, current knowledge, and future directions. Science and Engineering Ethics, 12, 53-74. https://doi.org/10.1007/PL00022268

Sterling, T. D. (1959). Publication Decisions and Their Possible Effects on Inferences Drawn from Tests of Significance--Or Vice Versa. Journal of the American Statistical Association, 54, 30. https://doi.org/10.2307/2282137

Sterne, J. A. C., \& Smith, G. D. (2001). Sifting the evidence-what's wrong with significance tests? Physical Therapy, 81, 1464-1469. https://doi.org/10.1093/ptj/81.8.1464

Stroebe, W., \& Strack, F. (2014). The Alleged Crisis and the Illusion of Exact Replication. Perspectives on Psychological Science, 9, 59-71. https://doi.org/10.1177/1745691613514450

van 't Veer, A. E., \& Giner-Sorolla, R. (2016). Pre-registration in social psychology-A discussion and suggested template. Journal of Experimental Social Psychology, 67, 2-12. https://doi.org/10.1016/j.jesp.2016.03.004

Wasserstein, R. L., Schirm, A. L., \& Lazar, N. A. (2019). Moving to a World Beyond “ $p$ \&lt; 0.05." The American Statistician, 73, 1-19. https://doi.org/10.1080/00031305.2019.1583913

Wicherts, J. M., \& Bakker, M. (2012). Publish (your data) or (let the data) perish! Why not publish your data too? Intelligence, 40, 73-76. https://doi.org/10.1016/j.intell.2012.01.004

Wicherts, J. M., Veldkamp, C. L. S., Augusteijn, H. E. M., Bakker, M., van Aert, R. C. M., \& van Assen, M. A. L. M. (2016). Degrees of Freedom in Planning, Running, Analyzing, and Reporting Psychological Studies: A Checklist to Avoid p-Hacking. Frontiers in Psychology, 7, 1832. https://doi.org/10.3389/fpsyg.2016.01832

Yates, F. (1951). The Influence of Statistical Methods for Research Workers on the Development of the Science of Statistics. Journal of the American Statistical Association, 46, 19-34. 
https://doi.org/10.1080/01621459.1951.10500764 